\title{
SOLUTION OF THE DUFFING EQUATION BY THE POWER SERIES METHOD
}

\author{
A. N. Pchelintsev ${ }^{1}$, S. Ahmad ${ }^{2}$ \\ Department of Higher Mathematics (1), \\ pchelintsev.an@yandex.ru; TSTU,Tambov,Russia; \\ Department of Mathematics and Statistics (2), \\ Zhengzhou University, Zhengzhou, Henan Province, China
}

Keywords: Duffing equation; power series method; nonlinear oscillator.

\begin{abstract}
In this article, the general form of Duffing equation is considered. The power series method is adapted to solve this equation with initial conditions. The convergence theorem of the method of power series is proved.
\end{abstract}

\section{Introduction}

Serious studies of forced nonlinear oscillators appeared early in the 20th century when Georg Duffing (1918) examined mechanical systems with nonlinearities. The Duffing equation is an outstanding nonlinear differential equation makes its presence in lot of problems like physical, engineering and even biological problems. The German electrical engineer Duffing introduced the model of Duffing equation in 1918.

The present article targets to offer an alternative technique of solution to the Duffing equation. By using the power series method, solutions of initial value problem for Duffing Equation were obtained in the form of infinite series. Schovanec and White used the Taylor series expansion method [1]. An analytical approach based on the power series method is proposed for the study of problems involving periodic motion. Qaisi has used the power series approach to solve undamped and unforced Duffing equation [2].

There are a number of computational methods that have been developed for solving the oscillatory problems of nonlinear oscillators, for example, the harmonic balance method, the variation iteration method, the homotopy perturbation method, the parameter-expanding method, the exp-function method, the differential transformmethod, and the optimal scale polynomial interpolation method, the Laplace decomposition methods were introduced by Khuri [3]. Chen [4] has used a target function method to solve Duffing equation. Yusufoglu numerically solved of Duffing equation by the Laplace decomposition algorithm [5]. Recently, Kumar has developed powerful method to solve time-fractional Fokker-Planck equation arising in solid state physics and circuit theory [6] and gas dynamics equation arising in shock fronts [7], telegraph equation via Laplace transform [8]. 
In our turn, in order to solve the Duffing equation we try to solve this equation by the power series method (PSM). PSM is a classical method to solve ordinary differential equations, which is closely related to the Taylor series method, but does not need an elaborate differential process to derive the expansion coefficients. However, the PSM is not presented as a general purpose algorithm, because it is necessary to derive the corresponding algebraic relation to generate the expansion coefficients case by case.

\section{Mathematical modeling}

In this section, we will define a non-linear second order differential equation with initial conditions:

$$
\begin{gathered}
\ddot{x}+\delta \dot{x}+\alpha x+\beta x^{3}=\gamma \cos \omega t ; \\
x(0)=p_{0} ; \dot{x}(0)=p_{1},
\end{gathered}
$$

where the values of $p_{0}$ and $p_{1}$ are set, $\delta$ is control the amount of damping, $\alpha$ is control the linear stiffness, $\beta$ is control the amount of non-linearity in the restoring force; if $\beta=0$ the Duffing equation is driven simple harmonic oscillator; $\gamma$ is the amplitude of the periodic driving force; if $\gamma=0$ the system is without a driving force; $\omega$ is angular frequency of the periodic driving force.

The power series of solutions for non-linear equations converge on a small intervals. Therefore the initial time must be transformed in the calculations. Let's write the problem in the general form as:

$$
\begin{gathered}
\ddot{x}+\delta \dot{x}+\alpha x+\beta x^{3}=\gamma \cos \left(\omega t+t_{0}\right) ; \\
x(0)=p_{0} ; \dot{x}(0)=p_{1},
\end{gathered}
$$

where $t_{0}$ is the initial time, $t$ is the time measured from the moment $t_{0}$,

$$
\cos \left(\omega t+t_{0}\right)=\sum_{i=0}^{\infty} \frac{\left.\left(\cos \left(\omega t+t_{0}\right)\right)^{(i)}\right|_{t=0}}{i !} t^{i}
$$

Let

$$
u_{0}=1, u_{1}=\frac{\omega u_{0}}{1}, u_{2}=\frac{\omega u_{1}}{2}, \ldots, u_{i}=\frac{\omega u_{i-1}}{i}, \ldots
$$

hence

$$
\cos \left(\omega t+t_{0}\right)=\sum_{i=0}^{\infty} u_{i} \cos \left(\omega t_{0}+\frac{\pi i}{2}\right) t^{i}
$$

\section{A modified power series method}

The power series method is a standard and effective tool for solving the Duffing equation. It gives the solution $x(t)$ in the form of a power series

$$
x(t)=\sum_{i=0}^{\infty} p_{i} t^{i} .
$$

Then

$$
\dot{x}(t)=\sum_{i=0}^{\infty}(i+1) p_{i+1} t^{i}=\sum_{i=0}^{\infty} q_{i} t^{i},
$$


where

$$
q_{i}=(i+1) p_{i+1} .
$$

Note that here

$$
q_{i+1}=(i+2) p_{i+2}
$$

Then

$$
\ddot{x}(t)=\left(\sum_{i=0}^{\infty} q_{i} t^{t^{\prime}}\right)^{\prime}=\sum_{i=0}^{\infty}(i+1) q_{i+1} t^{i}=\sum_{i=0}^{\infty}(i+1)(i+2) p_{i+2} t^{i} .
$$

Now taking square and cube of (3) we get the following results:

$$
\begin{gathered}
x^{2}=x \cdot x=\sum_{i=0}^{\infty}\left(\sum_{k=0}^{i} p_{k} p_{i-k}\right) t^{i}=\sum_{i=0}^{\infty} r_{i} t^{i} \\
x^{3}=x^{2} \cdot x=\sum_{i=0}^{\infty}\left(\sum_{j=0}^{i} r_{j} p_{i-j}\right) t^{i}=\sum_{i=0}^{\infty}\left[\sum_{j=0}^{i} p_{i-j} \sum_{k=0}^{j} p_{k} p_{j-k}\right] t^{i} .
\end{gathered}
$$

Now using (2) - (6) in equation (1), we get the following result

$$
p_{i+2}=-\frac{\delta p_{i+1}}{i+2}-\frac{\beta \sum_{j=0}^{i} p_{i-j} \sum_{k=0}^{j} p_{k} p_{j-k}}{(i+1)(i+2)}+\frac{\gamma u_{i} \cos \left(\omega t_{0}+\frac{\pi i}{2}\right)}{(i+1)(i+2)},
$$

for $i=0 \quad p_{2}$ is expressed through $p_{0}$ and $p_{1}$.

Note that $i$ ! grows faster than $\omega^{i}$, i.e.

$$
\lim _{i \rightarrow \infty} \frac{\omega^{i}}{i !}=0 .
$$

Then there exist such the value $i=i^{*}$, when

$$
\frac{\omega^{i}}{i !}<1
$$

for any $i>i^{*}$.

We denote by:

$$
\begin{gathered}
N_{\omega}=\max _{i=0, i^{*}} \frac{\omega^{i}}{i !} \geq 1 ; \frac{\omega^{0}}{0 !}=1 ; \\
\left|\cos \left(\omega t_{0}+\frac{\pi i}{2}\right)\right| \leq 1,\left|u_{i}\right| \leq N_{\omega} .
\end{gathered}
$$

Estimating the region of convergence of the power series is important when the integration step is selected.

Let $\Delta t$ is the integration step,

$$
\Delta t \in\left(-\frac{1}{h} ; \frac{1}{h}\right)
$$


$h$ is some number, which we also define:

$$
h_{1}=\max \left\{\left|p_{0}\right|,\left|p_{1}\right|, 1\right\} \geq 1 ; h_{2}=\delta+\beta\left(3 h_{1}^{2}+3 h_{1}+1\right)+\gamma N_{\omega}+1 \geq 1 ; h=h_{1} h_{2} \geq 1 .
$$

Theorem: The following inequalities are hold

$$
\left|p_{v}\right| \leq h^{v}
$$

where $v$ is any natural number.

Proof. We prove by the method of mathematical induction.

$\left|p_{1}\right| \leq h^{1}$ is obviously.

We assume that (8) is valid for $i=m+1, m$ is a natural number. Then it is also valid for any $n=\overline{1, i}$, that is

$$
\left|p_{n}\right| \leq h^{n}
$$

Note

$$
\begin{aligned}
& \sum_{j=0}^{i} p_{i-j} \sum_{k=0}^{j} p_{k} p_{j-k}=p_{i} p_{0}^{2}+p_{0}\left(2 p_{0} p_{i}+\sum_{k=1}^{i-1} p_{k} p_{i-k}\right)+\sum_{j=1}^{i-1} p_{i-j} \sum_{k=0}^{j} p_{k} p_{j-k}= \\
& =p_{i} p_{0}^{2}+p_{0}\left(2 p_{0} p_{i}+\sum_{k=1}^{i-1} p_{k} p_{i-k}\right)+\sum_{j=1}^{i-1} p_{i-j}\left(2 p_{0} p_{j}+\sum_{k=1}^{j-1} p_{k} p_{j-k}\right) .
\end{aligned}
$$

We estimate

$$
\begin{aligned}
& \left|\sum_{j=0}^{i} p_{i-j} \sum_{k=0}^{j} p_{k} p_{j-k}\right| \leq h_{1}^{2} h^{i}+h_{1}\left(2 h_{1} h^{i}+\sum_{k=1}^{i-1} h^{k} h^{i-k}\right)+\sum_{j=1}^{i-1} h^{i-j}\left(2 h_{1} h^{j}+\sum_{k=1}^{j-1} h^{k} h^{j-k}\right)= \\
& =h_{1}^{2} h^{i}+h_{1}\left(2 h_{1} h^{i}+h^{i}(i-1)\right)+2 h_{1} \sum_{j=1}^{i-1} h^{i}+\sum_{j=1}^{i-1} \sum_{k=1}^{j-1} h^{i}=h_{1}^{2} h^{i}+\left(2 h_{1}^{2}+(i-1) h_{1}\right) h^{i}+ \\
& +2 h_{1}(i-1) h^{i}+h^{i} \sum_{j=1}^{i-1}(j-1)=\left(3 h_{1}^{2}+(i-1) h_{1}\right) h^{i}+2 h_{1}(i-1) h^{i}+h^{i} \frac{0+(i-2)}{2}(i-1) \leq \\
& \leq\left(3 h_{1}^{2}+3 h_{1}(i+1)+(i+1)(i+2)\right) h^{i} \leq(i+1)(i+2)\left(3 h_{1}^{2}+3 h_{1}+1\right) h^{i} .
\end{aligned}
$$

Then from (7)

$$
\begin{gathered}
\left|p_{m+2}\right| \leq \frac{\delta\left|p_{m+1}\right|}{m+2}+\beta\left(3 h_{1}^{2}+3 h_{1}+1\right) h^{m}+\frac{\gamma\left|u_{m}\right|\left|\cos \left(\omega t_{0}+\frac{\pi m}{2}\right)\right|}{(m+1)(m+2)} \leq \delta h^{m+1}+ \\
+\beta\left(3 h_{1}^{2}+3 h_{1}+1\right) h^{m+1}+\gamma N_{\omega} h^{m+1} \leq\left(\delta+\beta\left(3 h_{1}^{2}+3 h_{1}+1\right)+\gamma N_{\omega}+1\right) h^{m+1}= \\
=h_{2} h^{m+1} \leq h_{1} h_{2} h^{m+1}=h^{m+2} .
\end{gathered}
$$

Hence proved that

$$
\left|p_{v}\right| \leq h^{v}
$$

where $v$ is any natural number.

The reported study was funded by RFBR for the research project No 20-01-00347. 


\title{
References
}

1. Schovanec L., White J.T. A Power Series Method for Solving Initial Value Problems Utilizing Computer Algebra Systems, International Journal of Computational Mathematics, 1993, vol. 47, issue 3-4, pp. 181-189.

2. Qaisi M.I. A Power Series Approach for the Study of Periodic Motion, Journal of Sound and Vibration, 1996, vol. 196, issue 4, pp. 401-406.

3. Khuri S.A. A Laplace Decomposition Algorithm Applied to a Class of Nonlinear Differential Equations, Journal of Applied Mathematics, 2001, vol. 1, issue 4, pp. 141-155.

4. Chen Y.Z. Solution of the Duffing Equation by Using Target Function Method, Journal of Sound and Vibration, 2002, vol. 256, issue 3, pp. 573-578.

5. Yusufoglu E. Numerical Solution of Duffing Equation by the Laplace Decomposition Algorithm, Applied Mathematics and Computation, 2006, vol. 177, no. 1, pp. 572-580.

6. Kumar S. Numerical Computation of Time-Fractional Fokker-Planck Equation Arising in Solid State Physics and Circuit Theory, Zeitschrift fur Naturforschung, 2013, vol. 68a, pp. 777-784.

7. Kumar S., Rashidi M.M. New Analytical Method for Gas Dynamics Equation Arising in Shock Fronts, Computer Physics Communications, 2014, vol. 185, issue 7, pp. 1947-1954.

8. Kumar S. A New Analytical Modelling for Telegraph Equation Via Laplace Transform, Applied Mathematical Modelling, 2014, vol. 38, issue 13, pp. 3154-3163.

\section{Решение уравнения Дуффинга методом степенных рядов}

\author{
А. Н. Пчелинцев ${ }^{1}$ С. Ахмад ${ }^{2}$ \\ Кафедра «Выстая математика» (1), \\ pchelintsev.an@yandex.ru; ФГБОУ ВО «ТГТУ», г.Тамбов, Россия; \\ кафедра математики и статистики (2), \\ Университет Чжэнчжоу, г. Чжэнчжоу, провинщия Хэнань, Китай
}

Ключевые слова: уравнение Дуффинга; метод степенных рядов; нелинейный осциллятор.

Аннотация: Рассмотрен общий вид уравнения Дуффинга. Метод степенных рядов адаптирован для решения данного уравнения с заданными начальными условиями. Доказана теорема о сходимости метода степенных рядов.

\section{Список литературы}

1. Schovanec, L. Power Series Method for Solving Initial Value Problems Utilizing Computer Algebra Systems / L. Schovanec, J. T. White // International Journal of Computational Mathematics. - 1993. - Vol. 47, Issue 3-4. - P. 181 - 189.

2. Qaisi, M. I. A Power Series Approach for the Study of Periodic Motion / M. I. Qaisi // Journal of Sound and Vibration. - 1996. - Vol. 196, Issue 4. - P. 401 - 406.

3. Khuri, S. A. A Laplace Decomposition Algorithm Applied to a Class of Nonlinear Differential Equations / S. A. Khuri // Journal of Applied Mathematics. 2001. - Vol. 1, Issue 4. - P. 141 - 155.

4. Chen, Y. Z. Solution of the Duffing Equation by Using Target Function Method / Y. Z. Chen // Journal of Sound and Vibration. - 2002. - Vol. 256, Issue 3. - P. 573 - 578. 
5. Yusufoglu, E. Numerical Solution of Duffing Equation by the Laplace Decomposition Algorithm / E. Yusufoglu // Applied Mathematics and Computation. 2006. - Vol. 177, no. 1. - P. $572-580$.

6. Kumar, S. Numerical Computation of Time-Fractional Fokker-Planck Equation Arising in Solid State Physics and Circuit Theory / S. Kumar // Zeitschrift fur Naturforschung. - 2013. - Vol. 68a. - P. 777 - 784.

7. Kumar, S. New Analytical Method for Gas Dynamics Equation Arising in Shock Fronts / S. Kumar, M. M. Rashidi // Computer Physics Communications. - 2014. Vol. 185, Issue 7. - P. 1947 - 1954.

8. Kumar, S. New Analytical Modelling for Telegraph Equation Via Laplace Transform / S. Kumar // Applied Mathematical Modelling. - 2014. - Vol. 38, Issue 13. P. $3154-3163$.

\section{Lösung der Duffing-Gleichung nach der Potenzreihenmethode}

Zusammenfassung: Die allgemeine Form der Duffing-Gleichung ist betrachtet. Die Potenzreihenmethode ist angepasst, um diese Gleichung unter gegebenen Anfangsbedingungen zu lösen. Das Theorem über die Konvergenz der Methode der Potenzreihen ist bewiesen.

\section{Résolution de l'équation de Duffing par la méthode des séries de puissance}

Résumé: Est considérée la vue générale de l'équation Duffing. La méthode des séries de puissance est adaptée pour la résolution d'une équation donnée avec des conditions initiales données. Est prouvé le théorème de convergence de la méthode des séries de puissance.

Авторы: Пчелинцев Александр Николаевич - кандидат физико-математических наук, доцент, заведующий кафедрой «Высшая математика», ФГБОУ ВО «ТГТУ», г. Тамбов, Россия; Ахммад Саджад - аспирант кафедры математики и статистики, Университет Чжэнчжоу, Чжэнчжоу, провинция Хэнань, Китай.

Рецензент: Жуковский Евгений Семёнович - доктор физико-математических наук, профессор, директор научно-исследовательского института математики, физики и информатики, ФГБОУ ВО «Тамбовский государственный университет имени Г. Р. Державина», г. Тамбов, Россия. 